\title{
THE MYSTERY OF THE DIFFERENCE IN THE LEVEL OF GENERAL TRUST IN CHINA AND JAPAN
}

\author{
Hiroki OZONO
}

Kagoshima University, Japan

\begin{abstract}
Previous studies show that the level of general trust in China is higher than in Japan, but the reason has not been explained sufficiently. In this study, I seek to explain this cultural difference in terms of trust among indirect friends and expectations of self-disclosure. I predicted that trust among indirect friends and expectations of self-disclosure would be higher in China, which would result in a higher level of general trust. Eighty-eight Chinese high school students and 63 Japanese undergraduate students completed my questionnaire. I replicated the cultural differences in the level of general trust. However, relational mobility, which explained cultural differences in the level of general trust in previous studies, did not explain my data. There were no cultural differences in trust among indirect friends, and expectations of self-disclosure were not higher in China. Ultimately, I failed to find an explanation for the cultural difference of general trust.
\end{abstract}

Key words: general trust, cultural differences, self-disclosure, network, relational mobility, mientzu

\section{INTRODUCTION}

General trust, or trust of people in general, is an important factor in sustaining and facilitating cooperation in a large society and has been widely investigated in the social sciences (Putnam, 1993; Fukuyama, 1995; Yamagishi, 1998/2011). In this study, I focus on the cultural difference in the level of general trust between China and Japan. Worldwide surveys have consistently shown that the Chinese tend to have a higher level of general trust than the Japanese. For example, in the World Values Survey (Wave 5, 2005-2009), the measure "most people can be trusted" was higher in China (36\%) than in Japan (9\%). The 2007 Pew Global Attitudes survey also showed the same trend in the measure "most people in society are trustworthy" (China: 79\%; Japan: 43\%; Wike \& Holzwart, 2008). Additionally, experimental studies using money, such as the trust game, also showed the same tendency; the Chinese are more likely to invest in others than the Japanese are despite the risk of betrayal (Takahashi et al., 2008; Li, 2011b). Conversely, both countries are in East Asia and share a common cultural background

I want to thank Masaki Yuki and Thomas Talhelm for giving me the Chinese version of the relational mobility scale, and I would like to thank Kosuke Takemura for giving me the original Japanese version of the "airport scenario." I would also like to thank Ro Ryu. This paper is based on his master thesis, and this study is filled with his original ideas.

Correspondence concerning this article should be addressed to Hiroki Ozono, Faculty of Law, Economics and Humanities, Kagoshima University, Japan (e-mail: hiroki.ozono@gmail.com). 
such as Confucianism and the use of Chinese characters. Various studies related to cultural psychology have assumed and reported similar psychological tendencies in both countries compared to the Western ones (e.g., Markus \& Kitayama, 1991; Nisbett, 2004). Thus, cultural differences in the level of general trust between two countries are outstanding and need to be explained.

The well-established argument explaining cultural differences in the level of general trust relies on differences in relational mobility (Yuki et al., 2007; Thomson et al., 2018). Relational mobility is defined as "the amount of opportunities people have in a given society or social context to select new relationship partners when necessary" (Yuki et al., 2007, p.1). Individuals in highly mobile societies are more likely to encounter opportunities to choose new relationships and can form and re-form them when needed. On the contrary, individuals in low-mobility societies tend to be firmly embedded in their social network, and it is more difficult for them to re-form relationships. Relational mobility is higher in the US than in Japan (Yuki et al., 2007; Thomson et al., 2018) and this difference can explain a wide variety of cultural differences in areas such as selfdisclosure (Schug, Yuki, \& Maddux, 2010), the emotion of shame (Sznycer et al., 2012), social phobias (Sato, Yuki, \& Norasakkunkit, 2014), and self-esteem and subjective wellbeing (Sato \& Yuki, 2014). Recently, Thomson et al. (2018) have shown that this argument can be applied not only to the differences between Japan and the US but that it can be adapted to explain differences between countries worldwide. Relational mobility also explains cultural differences in the level of general trust (Yamagishi, 1998/2011; Yamagishi \& Yamagishi, 1994; Yamagishi, Cook, \& Watabe, 1998, Yuki et al., 2007; Thomson et al., 2018). General trust serves to "emancipate" individuals from committed and closed relationships and form new relationships with outsiders (Yamagishi, 1998/2011). In high-mobility societies, this emancipating function is more important and beneficial than in low-mobility societies. Hence, people with high relational mobility are more likely to trust people in general (Yuki et al., 2007). While this argument is reasonable, both China and Japan are in East Asia, which is often categorized as a lowrelational mobility society (Thomson et al., 2018); thus, it is uncertain whether relational mobility can explain the differences between the two countries in the level of general trust.

There are direct arguments regarding the cultural differences between Japan and China (Takahashi et al., 2008; Li, 2011b). Researchers experimented using money (the trust game) with Chinese and Japanese participants and found that Chinese participants were more likely to invest in (trust) unknown others than the Japanese and to reciprocate their investment. Takahashi et al. (2008) explain these differences per different types of collectivism. In Japan, people tend to rely on assurance networks of well-established relationships where cheating on others would give one a bad reputation and would be punished by the members of one's social group. In other words, Japanese collectivism is defensive, and trust and reciprocal behavior are observed only within well-established social networks. Conversely, Chinese collectivism is more expansive. The Chinese tend to build up new social networks of guanxi (relationships; Hwang, 1998; Chou, Cheng, Huang, \& Cheng, 2006; Dunning \& Kim, 2007; Li, 2011a), and Dunning and Kim (2007) 
and Li (2011a) argued and demonstrated the expansive function of guanxi; thus, they are more likely to trust unknown people and to reciprocate trust. In this study, I investigate the reason for the difference in the level of general trust based on their argument. I focus on trust among indirect friends (e.g., a friend of a friend) and self-disclosure.

First, I focus on trust among indirect friends. Indirect friends are important in expanding one's relationships. $\mathrm{Li}$ (2011a) argued that expansibility (e.g., having a good relationship with a friend of a friend) is one of the important elements of guanxi. Hence, I hypothesized that trust among indirect friends would be higher in China than in Japan. In my study, I examine trust among second-order friends (a friend of a friend) and thirdorder friends (a friend of a second-order friend) as well as trust among first-order friends (one's own friend). I inferred that this expansion of trust, that is, trust among second-, third-, and higher-order friends, leads the Chinese to believe that "most people in society are trustworthy." This inference is consistent with the results of the World Values Survey about the trustworthiness of people you are meeting for the first time (strangers). In contrast to their high level of trust in most people, trust of strangers was low in China (unfortunately, this item was not included in the Japanese data). Complete strangers are outside of the expansion of friendships; in other words, outside of their own "society," thus the Chinese might not tend to trust them.

I also examined which factors facilitate trust among indirect friends. First, I thought that the Chinese might be more likely to regard indirect friends as their own friends to some extent. This belief in expanded friendship might lead to higher trust among indirect friends. Second, mientzu (face) might play an important role in facilitating trust among indirect friends. Mientzu is the concept of honor or reputation, and researchers often focus on mientzu to qualify Chinese culture (Bond \& Hwang, 1986; Chang \& Holt, 1994; Gao, 1998; Hwang, 1998). The Chinese tend to worry about their own mientzu and try to raise (not lower) their mientzu. Li (2011a) also argued that concern for reputation within social networks is one of the elements of guanxi. The Japanese language has a similar word and shares a similar concept, but concerns about mientzu among them might be weaker than among the Chinese. Sueda (1993) argued that the Chinese are concerned about mientzu more than the Japanese especially in the situation related to actual benefits. Therefore, when judging the trustworthiness of indirect friends, the Chinese might be more likely to assume that indirect friends would be concerned with their own mienztu and the mienztu of an intermediary(s); thus, they would be forced to be trustworthy. This assumption might lead to a higher level of trust among indirect friends in China than in Japan. To summarize, I predicted that belief in expanded friendship and concern for mientzu might facilitate a higher level of trust among indirect friends in China.

Additionally, I examine category-based and relationship-based trust (Yuki, Maddux, Brewer, \& Takemura, 2005). Yuki et al. (2005) argued that Westerners and Easterners use a different basis for judging the trustworthiness of unknown people. They demonstrated that Americans tend to trust people based on group category, so in-group members (e.g., a person who attends the same university) are more trusted than outgroup members (e.g., a person who attends a different university). In contrast, the Japanese tend to trust people based on direct or indirect relationships, so out-group 
members who have a potential indirect relationship (e.g., a person who attends a different university where you know someone) are more trusted by the Japanese than by Americans. This relationship-based trust is in line with the guanxi argument regarding China because guanxi is also the network of relationships. Therefore, I predicted that relationship-based trust would be observed in China as well as in Japan and the tendency toward relationship-based trust would probably be stronger in China.

The second point I focus on is self-disclosure. Previous studies have suggested that the Chinese tend to invest in others more vigorously than the Japanese and that they expand reciprocal relationships (Takahashi et al., 2008; Li, 2011b). Though the investment in their study was a financial one, this argument might be applied to the investment of personal information, in other words, self-disclosure. Self-disclosure is known to increase liking and intimacy with partners (Altman \& Taylor, 1973; Collins \& Miller, 1994; Laurenceau, Barrett, \& Pietromonaco, 1998). This is because selfdisclosure indicates a willingness to be vulnerable to others and signals trust and commitment (Mayer, Davis, \& Schoorman, 1995). I hypothesized that the Chinese are more likely to expect a high level of self-disclosure from unknown people than the Japanese to expand reciprocal relationships, which leads them to a higher level of general trust.

Previous studies have shown that Westerners are more likely to disclose themselves than East Asians (Chen, 1995; Asai \& Barnlund, 1998; Gudykunst \& Nishida, 1983; Kito, 2005; Ting-Toomey, 1991; Schug et al., 2010). Furthermore, several studies compare China and Japan directly (Gu, 2010; Hifumi, 2013). Hifumi (2013) revealed that the Chinese tend to disclose their emotions, personalities, and social roles more than the Japanese, which is consistent with my hypothesis. I investigated cultural differences in expectations of self-disclosure of positive, negative, and neutral information and how this influences the level of general trust. It should be noted that I investigated expectations of self-disclosure, not self-disclosure itself. This is because I believed that actual self-disclosure of yourselves does not always facilitate self-disclosure by others and so, expectations of self-disclosure are more important than actual self-disclosure to form the expectation of trustworthiness of unknown people, or general trust.

\section{Method}

\section{Participants}

Ninety-four Chinese high school students in Qingdao and sixty-four Japanese undergraduate students in Kagoshima answered the questionnaire during class voluntarily. Six Chinese and one Japanese participant had missing answers, and I excluded their data. Thus, the data of 88 Chinese (20 males and 68 females with a mean age of $17.02, S D=0.26)$ and 63 Japanese ( 45 males and 18 females with a mean age of $18.95, S D=0.72$ ) participants were used in the analyses.

\section{Procedure}

All participants from each country answered the questionnaire during class in under 15 minutes. After signing consent forms, participants completed a questionnaire, beginning with a short demographic form that requested their age, gender, and nationality. Each participant also completed several measures and questions 
(described later).

\section{Contents of the Questionnaire}

The questionnaire was constructed simultaneously in Chinese and Japanese; a back translation checked accuracy from the Japanese version by two Chinese graduate students who had lived in Japan for more than six years.

General trust. To confirm that the cultural differences in the level of general trust that had been reported in worldwide surveys would be replicated, two questions were asked: "Are most people in society trustworthy?" and "Can you trust a stranger?" The questions were answered using a 6-point scale ranging from 1 (strongly disagree) to 6 (strongly agree).

Relational mobility scale. Participants filled out the relational mobility scale (Yuki et al., 2007), which consists of 12 items asking participants to report their perceptions of relational mobility for people in their immediate environment (school, workplace, neighborhood, etc.) using a 6-point scale ranging from 1 (strongly disagree) to 6 (strongly agree). The items included questions like "They (i.e., people in my immediate social circle) have many chances to get to know other people." I received the Chinese version translated by Thomas Talhelm.

Trust among indirect friends. I asked participants to rate their level of trust in their friends and indirect friends. First, I asked them to imagine one of their best friends, called "A," and to rate how much they trust A on a 6-point scale ranging from 1 (not at all) to 6 (absolutely). Second, I asked them to imagine "B," who is one of the best friends of A and who is not the direct friend of the participants, and to rate B's trustworthiness on a 6-point scale. Third, participants were asked to imagine "C," who is one of the best friends of $\mathrm{B}$ and is neither the direct friend of A nor of the participants, and to rate C's trustworthiness in the same manner. I call A, B, and C first-order, second-order, and third-order friends, respectively.

After completing the questions, participants were asked how they felt when they rated the trustworthiness of B and C. I asked four questions for B and $\mathrm{C}$ each on a 6-point scale ranging from 1 (not at all) to 6 (absolutely). The four questions were as follows: "Did you think that $\mathrm{B} / \mathrm{C}$ was your own friend to some extent because $\mathrm{B} / \mathrm{C}$ is a friend of $\mathrm{A} / \mathrm{B}$ ?"; "Did you think that $\mathrm{B} / \mathrm{C}$ was concerned with the mientzu of the intermediary $\mathrm{A} / \mathrm{A}$ and $\mathrm{B}$ ?"; "Did you think that $\mathrm{B} / \mathrm{C}$ was worried about causing trouble for the intermediary A/A and B?"; and "Did you think that B/C was concerned with his or her own mientzu?" The first question was a measure of belief in expanded friendship, and the second, third, and fourth questions measured expectations of concerns regarding mientzu.

Relationship-based and category-based trust. To examine relationship-based and category-based trust (Yuki et al., 2005) in Japan and China, I asked the participants how to trust in-group, out-group with the possibility of a relationship, and out-group targets on a 6-point scale. In the study conducted by Yuki et al. (2005), three scenarios were used; however, I chose the scenario, called "airport scenario," in which they found the clearest results between Japan and the US to shorten my questionnaire. In this scenario, the participants imagine that they must ask a favor of an unknown passenger (the target) who is an in-group member (attending the same university), a person with whom they potentially have a relationship (attending a different university where you know someone), an out-group member (attending a different university), and a baseline passenger (complete stranger; see Yuki et al., 2005 for details). It should be noted that I asked the participants to answer all four conditions one after another, while these conditions were assigned to different participants in the study conducted by Yuki et al. (2005).

Expectations of self-disclosure. To examine expectations of self-disclosure by a stranger, the participants were asked to imagine a situation in which they talk with a stranger, a same-gender passenger who sits down next to them on the bus when they are traveling alone. I referred to the study by Sznycer et al. (2012) to create a framework for the situation. I asked the participants to check which items they thought that the passenger would disclose when asked. The items included address, telephone number, occupation, hometown, age, weight, height, medical history, academic background, income, family background, marriage history, occupations of family members, the amount in savings, prices of personal belongings, hobbies, favorite foods, place of work, and sexual orientation. Next, they were asked to rate how much they expected the passenger to disclose about their greatest success/failure if they asked him or her to rate it on a 6-point scale ranging from 1 (not at all) to 6 (absolutely). I asked what their expectations of self-disclosure regarding the greatest success/failure of their close friends would be as well, though there were no specific predictions for close friends. Finally, I asked the participants to rate their answers to the question "Do you 
think that trust in others strengthens as they share their personal information?" on a 6-point scale. This question measured belief in the role of self-disclosure in building trust, and I measured it to confirm my assumption regarding the function of self-disclosure.

\section{RESULTS}

I did not find any gender differences in the analyses including interactions, so I did not report the effect of gender on the latter.

\section{Are There Cultural Differences in the Level of General Trust?}

The means of general trust are shown in Table 1. I performed an independent t-test for two questions on general trust to examine the cultural differences. While the "trustworthiness of most people" was higher in China than Japan $(t(141.69)=4.53, p<$ $.001, d=0.73)$, there was no significant difference for the "trustworthiness of strangers" $(t(116.12)=0.90, p=.372, d=0.15)$. These tendencies are consistent with previous surveys (e.g., the World Values Survey). I use "trustworthiness of most people" only as the measure of general trust in the latter analysis because the purpose of this study is to investigate cultural differences.

\section{Does Relational Mobility Explain Cultural Differences in the Level of General Trust?}

First, I found that the relational mobility scale $(\alpha=.74)$ was higher in China $(M=$ $4.18, S D=0.66)$ than in Japan $(M=3.89, S D=0.60 ; t(140.40)=2.75, p=.007, d=$ 0.45). Relational mobility, however, was not correlated with general trust (the "trustworthiness of most people") in both countries ( $r=.14, p=.204$ in China; and $r=$ $-.08, p=.553$ in Japan). These results show that relational mobility did not explain the cultural differences between China and Japan.

\section{Are There Cultural Differences in the Level of Trust Among Indirect Friends?}

The means for the level of trust in first-order, second-order, and third-order friends (and attitudes towards the two levels of indirect friends) were calculated (see Table 2). To analyze the level of trust among direct and indirect friends, I conducted a 2 × 3 mixedfactorial analysis of variance (ANOVA) with country as the between-participant factor and friend type (first-order, second-order, and third-order) as a within-participant factor with the trustworthiness of each as the dependent variable. The results revealed a significant main effect for friend type $\left(F(1,298)=422.62, p<.001, \eta_{p}{ }^{2}=.739\right)$, but the main effect for the country and the interaction was not significant $(F(1,149)=0.02, p=$

Table 1. Level of general trust in China and Japan (standard deviations in parentheses)

\begin{tabular}{lll}
\hline & China & Japan \\
\hline Trustworthiness of most people & $3.64(1.29)$ & $2.73(1.15)$ \\
Trustworthiness of strangers & $2.53(0.79)$ & $2.67(0.97)$ \\
\hline
\end{tabular}


Table 2. Level of trust in first-order, second-order, and third-order friends (standard deviations in parentheses)

\begin{tabular}{lcc}
\hline & China & Japan \\
\hline Trust in first-order friend (A) & $5.15(1.09)$ & $5.24(0.73)$ \\
Trust in second-order friend (B) & $3.38(1.41)$ & $3.38(1.05)$ \\
Trust in third-order friend (C) & $2.59(1.19)$ & $2.43(1.07)$ \\
\hline Attitudes towards B & & \\
\hline B is my own friend to some extent & $3.30(1.70)$ & $2.60(1.45)$ \\
B is concerned with the mientzu of the intermediary A & $3.19(1.56)$ & $2.43(1.32)$ \\
B is concerned about causing trouble for the intermediary A & $3.23(1.56)$ & $2.48(1.24)$ \\
B is concerned about his/her own mientzu & $3.08(1.59)$ & $2.38(1.21)$ \\
\hline Attitudes towards C & & \\
\hline C is my own friend to some extent & & \\
C is concerned about the mientzu of the intermediaries A and B & $2.83(1.59)$ & $1.92(1.14)$ \\
C is concerned about causing trouble for the intermediaries A and B & $2.80(1.61)$ & $2.00(1.15)$ \\
C is concerned about his/her own mientzu & $2.93(1.65)$ & $2.10(1.24)$ \\
\hline
\end{tabular}

$\left..885, \eta_{p}^{2}=.000 ; F(1,298)=0.93, p=.383, \eta_{p}^{2}=.006\right)$. The multiple comparison (Holm method) for friend type showed that first-order friends were trusted more than second-order friends $(t(149)=18.30, p<.001, d=1.58)$ and second-order friends were trusted more than third-order friends $(t(149)=11.94, p<.001, d=0.71)$. Thus, I did not find any cultural differences in the level of trust in indirect friends.

Next, I examined how beliefs about expanded friendship and mientzu affect perceptions of the trustworthiness of an indirect friend. All measures of attitudes regarding the judgment of trustworthiness were significantly different between cultures: the Chinese tended to think of second-order and third-order friends as their own friends more than the Japanese did and the Chinese tended to think that second-order and thirdorder friends would be concerned about the intermediary's mienztu, troubles, and their own mienztu more than the Japanese did $(2.61<t s<4.09$, ps $<0.05)$. These tendencies were consistent with my predictions. Additionally, correlations among these measures were calculated (see Table 3). The measures regarding mientzu are strongly or modestly correlated with each other. The correlation between the belief of expanded friendship and concerns about mientz $u$ looks stronger in China than in Japan. While the results are intriguing, the finding is beyond my research question; thus, I do not discuss it further.

I performed multiple regression analyses to examine how these beliefs affected perceptions of the trustworthiness of indirect friends (see Table 4). To avoid multicollinearity due to strong correlation, the measure of " $\mathrm{B} / \mathrm{C}$ is concerned about 
Table 3. Correlations among attitudes towards second-order and third-order friends (Chinese data/ Japanese data)

\begin{tabular}{|c|c|c|c|c|}
\hline Attitudes towards B & & 1 & 2 & 3 \\
\hline \multicolumn{5}{|l|}{$1 \mathrm{~B}$ is my own friend to some extent } \\
\hline $2 \mathrm{~B}$ is concerned with the mientzu of the intermediary A & .061 & /-.045 & & \\
\hline $\begin{array}{l}3 \mathrm{~B} \text { is concerned about causing trouble for the } \\
\text { intermediary A }\end{array}$ & .113 & / -.063 & $.558 * * / .603 * *$ & \\
\hline $4 \mathrm{~B}$ is concerned about his/her own mientzu & .128 & $/-.050$ & $.519 * * / .270 *$ & $.490 * * / .499 * *$ \\
\hline Attitudes towards C & & 5 & 6 & 7 \\
\hline \multicolumn{5}{|l|}{$5 \mathrm{C}$ is my own friend to some extent } \\
\hline $\begin{array}{l}6 \mathrm{C} \text { is concerned about the mientzu of the intermediaries } \\
\mathrm{A} \text { and } \mathrm{B}\end{array}$ & \multicolumn{3}{|c|}{$.326 * * / .155$} & \\
\hline $\begin{array}{l}7 \mathrm{C} \text { is concerned about causing trouble for the } \\
\text { intermediaries } \mathrm{A} \text { and } \mathrm{B}\end{array}$ & .248 & $* / .094$ & $.888 * * / .824 * *$ & \\
\hline $8 \mathrm{C}$ is concerned about his/her own mientzu & $.277^{*}$ & $* * / .044$ & $.584 * * / .439 * *$ & $.530 * * / .622 * *$ \\
\hline
\end{tabular}

Note. $* p<.05, * * p<.01$

Table 4. Multiple regression analyses $(\mathrm{DV}=$ trust in second/third order friend).

\begin{tabular}{|c|c|c|c|c|}
\hline & \multicolumn{2}{|c|}{ Second order friend } & \multicolumn{2}{|c|}{ Third-order friend } \\
\hline & Step 1 & Step2 & Step1 & Step2 \\
\hline Intercept & $3.25 * *$ & $3.25 * *$ & $2.47 * *$ & $2.48 * *$ \\
\hline Culture (0: China; 1 : Japan) & 0.31 & 0.30 & 0.12 & 0.14 \\
\hline Belief in expanded friendship & $0.32 * *$ & $-0.34 * *$ & $0.26 * *$ & $0.22 * *$ \\
\hline Concerns about mientzu of the intermediary(s) & 0.08 & -0.05 & 0.09 & 0.05 \\
\hline Concerns about one's own mientzu & 0.04 & 0.15 & 0.06 & 0.12 \\
\hline Culture $\mathrm{x}$ belief in expanded friendship & & -0.07 & & 0.13 \\
\hline Culture $\mathrm{x}$ concerns about mientz $u$ of the intermediary(s) & & $0.31 *$ & & 0.12 \\
\hline Culture $\mathrm{x}$ concerns about one's own mientzu & & -0.29 & & -0.17 \\
\hline$R^{2}$ & $.18^{* *}$ & $.21^{* *}$ & $.17 * *$ & $.18^{* *}$ \\
\hline
\end{tabular}

Note. ${ }^{*} p<.05, * * p<.01$

causing trouble for the intermediary A/A and B" was excluded from the analysis, and the quantitative variables were centered. The results show that belief in expanded friendship (e.g., a friend of my friend is to some extent my own friend) positively affects trust among indirect friends, but belief in concerns about mientzu did not affect trust among 
them. Even when I add culture $\mathrm{x}$ beliefs interaction factors, there are no significant interactions except for a belief in being concerned about the mientzu of intermediary in second-order friends. In conclusion, though I hypothesized that belief in expanded friendship and mientzu would facilitate trust among indirect friends, these results were inconsistent with this hypothesis, especially for mientzu.

\section{Relationship-Based or Category-Based Trust?}

I analyzed the level of trust in the target in the airport scenario, which clarified relationship-based and category-based trust (Yuki et al., 2005). The results are shown in Table 5. I conducted a $2 \times 4$ mixed-factorial ANOVA with country as the betweenparticipant factor and target type (in-group, potential relationship, out-group, and baseline) as a within-participant factor with the level of trust in each as the dependent variable. The results revealed a significant main effect for culture $(F(1,149)=30.69, p$ $\left.<.001, \eta_{p}{ }^{2}=.171\right)$; the Chinese trusted a target more than the Japanese did. Additionally, I found a significant main effect for target type $(F(1,447)=167.88, p<$ $\left..001, \eta_{p}{ }^{2}=.530\right)$ and interaction $\left.F(1,447)=15.66, p<.001, \eta_{p}{ }^{2}=.095\right)$, so I performed multiple comparisons (Holm method) in China and Japan separately. In China, the ingroup target was more trusted than the potential relationship target, the potential relationship target was more trusted than the out-group target, and the out-group target was more trusted than the baseline target (in-group versus potential relationship, $t(149)=$ 13.23, $p<.001, d=1.53$; potential relationship versus out-group, $t(149)=4.78, p<$ $.001, d=0.31$; out-group versus baseline, $t(149)=6.47, p<.001, d=0.46$ ). In Japan, I found the same trends-in-group versus potential relationship, $t(149)=5.17, p<.001, d$ $=0.53$; potential relationship versus out-group, $t(149)=3.46, p=.001, d=0.26$; outgroup versus baseline, $t(149)=2.72, p=.007, d=0.23$. Thus, I found significant differences among all factors, and it is difficult to discuss the difference between China and Japan. However, the pattern of China is closely linked to category-based trust rather than relationship-based trust, because in China, the effect size between the in-group and potential relationship categories is very large $(d=1.53)$, but the one between the potential relationship and out-group category is medium-sized $(d=0.31)$. On the contrary, it is difficult to classify the pattern of the Japanese into a category- or relationship-based trust partly because of floor effect in Japan. Though I predicted that relationship-based trust would be stronger in China than in Japan, the results were

Table 5. Trust in targets in the "airport scenario" (standard deviations in parentheses)

\begin{tabular}{lcc}
\hline & China & Japan \\
\hline In-group & $4.15(1.12)$ & $2.62(1.29)$ \\
Potential relationship & $2.81(1.19)$ & $2.00(1.08)$ \\
Out-group & $2.45(1.23)$ & $1.70(1.04)$ \\
Baseline & $1.94(1.17)$ & $1.44(0.93)$ \\
\hline
\end{tabular}


inconsistent with this prediction.

Are Expectations of Self-Disclosure Higher in China?

To analyze the expectations of self-disclosure of neutral information, I counted how many items each participant checked. The average number in China $(M=5.11, S D=$ 2.66) was lower than in Japan $(M=6.03, S D=2.67$; Mann-Whitney U test, $p<.001)$, which was the opposite of what I predicted in my hypothesis. I also analyzed the differences for each item. The Japanese significantly expected that others would disclose their hometown (China: 67\%; Japan: 94\%, $p<.001$ ), age (China: 60\%; Japan: 78\%, $p=$ .034 ), weight (China: 5\%; Japan: 19\%, $p=.006$ ), height (China: 22\%; Japan: 38\%, $p=$ .003 ), and family background (China: 15\%; Japan: 44\%, $p<.001$ ) as opposed to the Chinese, who expected that others would disclose the price of personal belongings (China: 16\%; Japan: $5 \%, p=.005$ ). As for weight, the difference might be due to the greater number of female participants in China.

Next, I performed 2 (culture: China/Japan) x 2 (experience: success/failure) x 2 (relationship: stranger/close friend) mixed factorial ANOVA (the means are shown in Fig. 1). The results revealed a significant main effect for experience $(F(1,149)=$ 171.27, $\left.p<.001, \eta_{p}{ }^{2}=.535\right)$, a significant main effect for relationship $(F(1,149)=$ $\left.165.81, p<.001, \eta_{p}{ }^{2}=.527\right)$, and non-significance for culture $(F(1,149)=0.94, p=$ $\left..334, \eta_{p}{ }^{2}=.006\right)$. The participants expected that others would be more likely to disclose a successful experience, and a close friend was expected to disclose his or her own experiences more often than a stranger was. Furthermore, there was a culture $\mathrm{x}$ experience interaction $\left(F(1,149)=49.43, p<.001, \eta_{p}{ }^{2}=.249\right)$. The test of the simple main effect showed that the Chinese tended to expect others to disclose successful experiences more than the Japanese $\operatorname{did}\left(F(1,298)=11.60, p<.001, \eta_{p}^{2}=.038\right)$, but

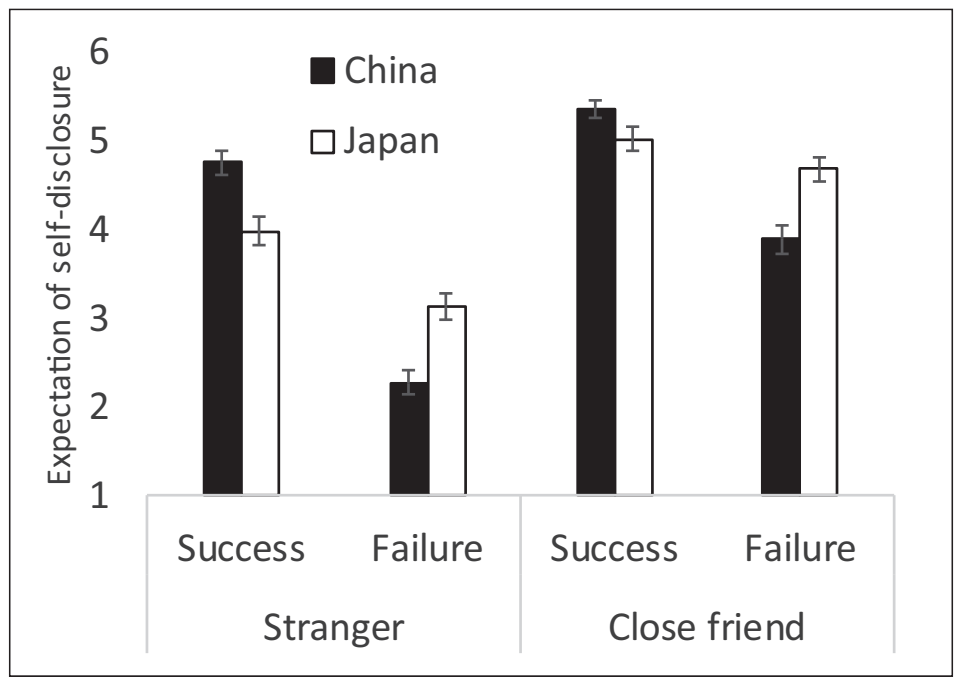

Fig. 1. Expectations of self-disclosure by a stranger and a close friend (error bars are standard errors) 
that the Japanese tended to expect others to disclose failure more than the Chinese did ( $F$ $\left.(1,149)=24.62, p<.001, \eta_{p}{ }^{2}=.076\right)$. This tendency was not significantly different by relationship because the three-way interaction of culture $\mathrm{x}$ experience $\mathrm{x}$ relationship was not significant $\left(F(1,149)=2.69, p=.103, \eta_{p}{ }^{2}=.018\right)$. I also found an experience $\mathrm{x}$ relationship interaction $\left(F(1,149)=27.51, p>.001, \eta_{p}{ }^{2}=.156\right)$, but this was not related to my hypothesis, so I have chosen not to discuss it. These results suggest that the Chinese expect others to disclose their own information more than the Japanese only where positive information is concerned, but the Japanese tend to expect others to disclose neutral or negative information more often than the Chinese.

I analyzed the correlations between general trust and expectations of self-disclosure by strangers, and I found a weak positive correlation between general trust and expectations of self-disclosure of negative experiences both in China $(r=.184, p=.087)$ and Japan $(r=.325, p=.009)$. These results are consistent with my assumption, that is, expectations of self-disclosure enhance general trust. As for positive experiences and neutral information, however, I did not find any positive correlations $(-.090<r s<.152$, $p s>.10)$. Finally, I found a significant difference in the belief in the importance of selfdisclosure in building trust. I asked a question, "do you think that trust in others strengthens as they share their personal information?" and found that this belief was weaker in China $(M=2.77, S D=1.52)$ than in Japan $(M=3.46, S D=1.19 ; t(147.81)=$ $3.11, p=.002, d=0.49)$. I did not expect this cultural difference.

\section{Discussion}

First, I examined the cultural difference in the level of general trust between China and Japan, and I found that the perception of trustworthiness in most people was higher in China, but there was no difference in the perception of trustworthiness in strangers. I reproduced the findings in previous studies. Because the participants in my study were limited to Chinese high school students and Japanese undergraduate students in local cities, the representativeness of the samples might be limited and might not reflect the general Chinese and Japanese populations. However, I should emphasize that the replication of general trust in my study ensures the premise of my discussion.

Relational mobility did not explain the cultural differences in general trust in my data. This means that other factors besides relational mobility must be considered to understand the difference between China and Japan in general trust. I should note that relational mobility was higher in China than in Japan on average. This means that "macro-level" differences, not "micro-level" (individual) differences, of relational mobility might enable us to explain the difference in the level of general trust (Thomson et al., 2018) between the two countries. Though further study will be necessary, it should be emphasized that I failed to replicate the results of Yuki et al. (2007) which explain the difference in the level of general trust between Japan and the US in terms of individuallevel relational mobility.

There was no cultural difference in trust among indirect friends, which was 
inconsistent with my hypothesis. I found consistent results concerning belief in expanded friendship and concerns regarding mientzu; the Chinese are more likely to regard secondand third-order friends as their friends, and they expected more concerns regarding mientzu from the second- and third-order friend him/herself and the intermediary(s) than the Japanese did. However, belief in mientzu did not explain trust in indirect friends. Though belief in expanded friendship explained the trust among indirect friends, there was no difference in the level of trust in indirect friends between Japan and China, as mentioned. These results mean that general trust in China is constructed with neither relation to expanded friendship nor mientzu, which are two factors characterizing relationships in China. Furthermore, the results of the "airport scenario," which classified relation-based and category-based trust, suggest that the data pattern of China was classified as category-based trust rather than relationship-based trust. The Japanese data have a floor effect and are difficult to discuss. While I cannot directly compare my results with those of the previous study by Yuki et al. (2005) due to several methodological differences, such as within-participant design (my study) and betweenparticipant design (their study), it should be emphasized that the Chinese data were closed to category-based trust, and this pattern was opposite to the framework of relationships in China, which I hypothesized. In conclusion, I failed to support my idea, that is, the expansion of relationships in China can explain higher trust to most people in society (general trust).

As for expectations of self-disclosure, the results were inconsistent with my hypothesis. While the Chinese tended to expect higher self-disclosure than the Japanese for positive information (successful experiences), they had lower expectations for negative experiences (failures) and neutral information. These results mean that the Chinese expect others to selectively disclose their own information in a way that raises their reputation. The root of this manipulative self-disclosure might be a strong concern for mientzu in China. Conversely, the Japanese might have a more self-effacing tendency to adapt to their embedded group (Yamagishi et al., 2012) than the Chinese, which leads the Japanese to disclose their negative experiences. From this perspective, it is understandable that belief in the function of self-disclosure in building trust is lower in China than in Japan. Self-disclosure might be perceived as a tool for self-betterment and is sometimes manipulated in China; thus, such information might be less useful in strengthening trust with others in China than in Japan.

\section{Conclusion}

While I replicated the higher level of general trust in China than in Japan, neither relational mobility, trust among indirect friends, mienztu, nor expectations of selfdisclosure could explain this cultural difference. I should discuss the reason. In the studies by Takahashi et al. (2008) and Li (2011b), which clearly showed the cultural difference using the trust game, the measure of trust was financial investment, and reciprocity was expected. Especially, Li (2011b) revealed that the cultural difference of trust between China and Japan disappeared when participants could not expect reciprocity using the "faith game" (for a detailed explanation of the faith game, see 
Kiyonari, Yamagishi, Cook, \& Cheshire, 2006). Hence, they concluded that high trust in strangers in China depends upon an investment in building new reciprocal relationships. On the contrary, the points of financial investment and the expectation of reciprocity were not present in my study of trust among indirect friends. In my study, I only asked about trust among indirect friends without any specific context. This might be the reason that I failed to find a higher level of trust in indirect friends in China. Though this explanation is plausible to some extent, this does not fully explain the cultural differences in the level of general trust and trust in the targets in the "airport scenario" in my study. This is because the question of general trust is very abstract and the "airport scenario" also lacked the elements of financial investment and the expectation of reciprocity. Nevertheless, I found a higher level of trust in China than in Japan in these questions. Additionally, it is difficult to explain why the Chinese were closed to category-based trust rather than relationship-based trust.

As for expectations of self-disclosure, I consider that manipulative self-disclosure due to concerns for mientzu might reduce the reliability of self-disclosure in China. Therefore, unlike financial investment, active investment of self-disclosure is not facilitated in China, so that self-disclosure might not function to build trust in others.

My study had several limitations. First, the population of my sample was very limited. While I could replicate the cultural differences in the level of general trust, whether my results could be generalized is still in question. Particularly, my Chinese samples were high school students in Qingdao, and my Japanese samples were undergraduate students in Kagoshima. Thus, the factors of age and education level are confounded with the factor of culture. Additionally, Qingdao is a major urban center city whereas Kagoshima is a medium-size regional city, which might influence the results. I should use more representative samples to confirm the validity of my results. Second, I should pay more attention to differences in language. For example, the meaning of mientzu might not be the same between the two countries, which might have influenced the results. Third, I used responses from the measures without establishing their measurement equivalence between China and Japan. The measurements should be refined more. Fourth, it is necessary to discuss the impact of other factors. For example, the Hukou (family register) system in China discourages movements of people, especially from rural to urban areas (Chan \& Zhang, 1999), which might influence relational mobility in China.

In conclusion, interesting mysteries remain regarding cultural differences in the level of general trust between China and Japan. Unfortunately, I could not solve this mystery fully, or I might expand the mystery. I believe, however, that negative results can be important in advancing scientific inquiry. Thus, future investigations are expected.

\section{REFERENCES}

Altman, I., \& Taylor, D. A. (1973). Social penetration: The development of interpersonal relationships. 
New York, NY: Holt, Rinehart \& Winston.

Asai, A., \& Barnlund, D. C. (1998). Boundaries of the unconscious, private, and public self in Japanese and Americans: A cross-cultural comparison. International Journal of Intercultural Relations, 22, 431-452.

Bond, M. H., \& Hwang, K. K. (1986). The social psychology of Chinese people. In M. H. Bond (Ed.), The psychology of Chinese people (pp.213-266). New York, NY: Oxford University Press.

Chan, K. W., \& Zhang, L. (1999). The hukou system and rural-urban migration in China: Processes and changes. The China Quarterly, 160, 818-855.

Chang, H. C., \& Holt, G. R. (1994). A Chinese perspective on face as inter-relational concern. In S. TingToomey (Ed.), The challenge of facework: Cross-cultural and interpersonal issues (pp. 95-132). Albany, NY: State University of New York.

Chen, G. (1995). Differences in self-disclosure patterns among Americans versus Chinese: A comparative study. Journal of Cross-Cultural Psychology, 26, 84-91.

Chou, L. F., Cheng, B. S., Huang, M. P., \& Cheng, H. Y. (2006). Guanxi networks and members' effectiveness in Chinese work teams: Mediating effects of trust networks. Asian Journal of Social Psychology, 9, 79-95.

Collins, N. L., \& Miller, L. C. (1994). Self-disclosure and liking: A meta-analytic review. Psychological Bulletin, 116, 457-475.

Dunning, J. H., \& Kim, C. (2007). The cultural roots of guanxi: An exploratory study. World Economy, 30, $329-341$.

Fukuyama, F. (1995). Trust: The social virtues and the creation of prosperity. Glencoe, IL: Free Press.

Gao, G. (1998). An initial analysis of the effects of face and concern for "other" in Chinese interpersonal communication. International Journal of Intercultural Relations, 22, 467-482.

$\mathrm{Gu}$, P. (2010). Chugoku to nihon no daigakusei no taijin kankei ni okeru jiko-kaiji no arikata ni kansuru hikaku kenkyu [A cross-cultural study of ideal self-disclosure method in the personal relationships between Chinese and Japan university students]. Kyushu University Psychological Research, 11, 153-163.

Gudykunst, W. B., \& Nishida, T. (1983). Social penetration in Japanese and American close friendships. In R. N. Bostrom \& B. H. Westley (Eds.), Communication yearbook 7 (pp. 592-610). Beverly Hills, CA: Routledge.

Hifumi, T. (2013). Joshi gakusei ni yoru jiko-kaiji no kaiji naiyou-men to shinri-men ni kansuru nicchu taisho kenkyu [A contrastive study of self-disclosure in Japanese and Chinese female students: Selfdisclosure categories and the psychological process]. Bungei Gengo Kenkyu, 64, 75-94.

Hwang, K. K. (1998). Guanxi and mientze: Conflict resolution in Chinese society. Intercultural Communication Studies, 7, 17-38.

Kito, M. (2005). Self-disclosure in romantic relationships and friendships among American and Japanese college students. The Journal of Social Psychology, 145, 127-140.

Kiyonari, T., Yamagishi, T., Cook, K. S., \& Cheshire, C. (2006). Does trust beget trustworthiness? Trust and trustworthiness in two games and two cultures: A research note. Social Psychology Quarterly, 69, $270-283$.

Laurenceau, J., Barrett, L. F., \& Pietromonaco, P. R. (1998). Intimacy as an interpersonal process: The importance of self-disclosure, partner disclosure, and perceived partner responsiveness in interpersonal exchanges. Journal of Personality and Social Psychology, 74, 1238-1251.

Li, Y. (2011a). Chugoku-jin no Guanxi ninchi to Guanxi shakudo no sakusei [Development of a scale to measure guanxi in China] (Working paper No. 124). Hokkaido University, Center for Experimental Research in Social Sciences.

Li, Y. (2011b). Bunpai inin gemu ni okeru sinrai kodo no nicchu hikaku [Comparison of trust behaviors in the faith game between Japan and China] (Working paper No. 125). Hokkaido University, Center for Experimental Research in Social Sciences.

Markus, H. R., \& Kitayama, S. (1991). Culture and the self: Implications for cognition, emotion, and motivation. Psychological Review, 98, 224-253.

Mayer, R. C., Davis, J. H., \& Schoorman, F. D. (1995). An integrative model of organizational trust. Academy of Management Review, 20, 709-734.

Nisbett, R. (2004). The geography of thought: How Asians and Westerners think differently... and why. New 
York, NY: Simon \& Schuster.

Putnam, R. D. (1993). The prosperous community. The American Prospect, 4, 35-42.

Sato, K., \& Yuki, M. (2014). The association between self-esteem and happiness differs in relationally mobile vs. stable interpersonal contexts. Frontiers in Psychology, 5, 1113.

Sato, K., Yuki, M., \& Norasakkunkit, V. (2014). A socio-ecological approach to cross-cultural differences in the sensitivity to social rejection: The partially mediating role of relational mobility. Journal of Cross-Cultural Psychology, 45, 1549-1560.

Schug, J., Yuki, M., \& Maddux, W. (2010). Relational mobility explains between- and within-culture differences in self-disclosure to close friends. Psychological Science, 21, 1471-1478.

Sueda, K. (1993). Chugokujin ga motsu mentsu no gainen to nihonjin tono komyunikeshon [Chinese perception of Face (Mianzi) and its influence on communicating with the Japanese]. Annual Review of Sociology, 1993, 191-202.

Sznycer, D., Takemura, K., Delton, A. W., Sato, K., Robertson, T., Cosmides, L., \& Tooby, J. (2012). Cross-cultural differences and similarities in proneness to shame: An adaptationist and ecological approach. Evolutionary Psychology, 10. doi: 10.1177/147470491201000213

Takahashi, C., Yamagishi, T., Liu, J. H., Wang, F., Lin, Y., \& Yu, S. (2008). The intercultural trust paradigm: Studying joint cultural interaction and social exchange in real time over the Internet. International Journal of Intercultural Relations, 32, 215-228.

Thomson, R., Yuki, M., Talhelm, T., Schug, J., Kito, M., Ayanian, A. H., . . . Visserman, M. L. (2018). Relational mobility predicts social behaviors in 39 countries and is tied to historical farming and threat. Proceedings of the National Academy of Sciences, 115, 7521-7526.

Ting-Toomey, S. (1991). Intimacy expressions in three cultures: France, Japan, and the United States. International Journal of Intercultural Relations, 15, 29-46.

Wike, R., \& Holzwart, K. (2008). Where trust is high, crime and corruption are low. Pew Global Attitudes Project, Pew Research Center Publication.

Yamagishi, T. (2011). Trust: The evolutionary game of mind and society. New York, NY: Springer Science \& Business Media. (Original work published 1998)

Yamagishi, T., Cook, K. S., \& Watabe, M. (1998). Uncertainty, trust, and commitment formation in the United States and Japan. American Journal of Sociology, 104, 165-194.

Yamagishi, T., Hashimoto, H., Cook, K. S., Kiyonari, T., Shinada, M., Mifune, N., . . . Li, Y. (2012). Modesty in self-presentation: A comparison between the USA and Japan. Asian Journal of Social Psychology, 15, 60-68.

Yamagishi, T., \& Yamagishi, M. (1994). Trust and commitment in the United States and Japan. Motivation and Emotion, 18, 129-166.

Yuki, M., Maddux, W. W., Brewer, M. B., \& Takemura, K. (2005). Cross-cultural differences in relationship- and group-based trust. Personality and Social Psychology Bulletin, 31, 48-62.

Yuki, M., Schug, J., Horikawa, H., Takemura, K., Sato, K., Yokota, K., \& Kamaya, K. (2007). Development of a scale to measure perceptions of relational mobility in society (Working paper No. 75). Hokkaido University, Center for Experimental Research in Social Sciences. 